PROFESIONALES Y HERRAMIENTAS PARA EL DESARROLLO LOCAL Y SUS SINERGIAS TERRITORIALES. EVALUACIÓN Y PROPUESTAS DE FUTURO IX Coloquio Nacional de Desarrollo Local del GTDL-AGE 

ANTONIO MARTÍNEZ PUCHE, XAVIER AMAT MONTESINOS, ISABEL SANCHO CARBONELL y DANIEL SANCHIZ CASTAÑO (EDS.)

\section{PROFESIONALES Y HERRAMIENTAS PARA EL DESARROLLO LOCAL Y SUS SINERGIAS TERRITORIALES. EVALUACIÓN Y PROPUESTAS DE FUTURO}

IX Coloquio Nacional de Desarrollo Local del GTDL-AGE

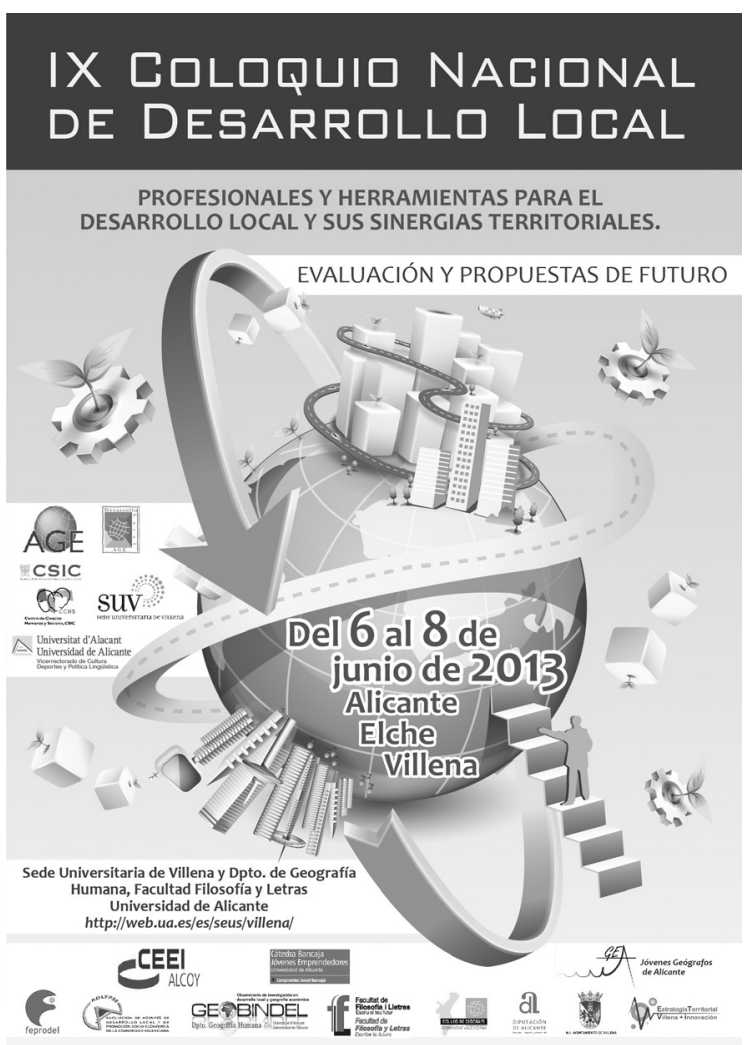


Este libro ha sido examinado y valorado por evaluadores ajenos a la Universidad de Alicante, con el fin de garantizar la calidad científica del mismo.

Publicacions de la Universitat d'Alacant

03690 Sant Vicent del Raspeig

Publicaciones@ua.es

http://publicaciones.ua.es

Telèfon: 965903480

(C) Antonio Martínez Puche, Xavier Amat Montesinos,

Isabel Sancho Carbonell y Daniel Sanchiz Castaño (eds.), 2016

(C) d'aquesta edició: Universitat d'Alacant

ISBN: 978-84-16724-00-0

Dipòsit legal: A 92-2016

Disseny de coberta: candela ink

Composició: Página Maestra (Miguel Ángel Sánchez Hernández)

Impressió i enquadernació: Guada Impresores

\section{unte \\ Unión de Editoriales
Universitarias Españolas \\ WWW.une.es
WWA}

Esta editorial es miembro de la UNE, cosa que garantiza la difusión y comercialización nacional y internacional de sus publicaciones.

Reservados todos los derechos. Cualquier forma de reproducción, distribución, comunicación pública o transformación de esta obra sólo puede ser realizada con la autorización de sus titulares, salvo excepción prevista por la ley. Diríjase a CEDRO (Centro Español de Derechos Repográficos, www.cedro.org) si necesita fotocopias o escanear algún fragmento de esta obra. 


\title{
INICIATIVAS JUVENILES COMO BASE DE DESARROLLO INTEGRAL. ESPACIOS DE CO-WORKING Y NUEVAS ECONOMÍAS: YOUTH HUB
}

\author{
Jorge Galán Soler \\ Gruppo d'Azione Locale (Eloro) \\ jorge.galansoler@gmail.com \\ Alberto Lorente Saiz. \\ Gruppo d'Azione Locale (Eloro) \\ albertolorentesaiz@gmail.com
}

\section{RESUMEN}

En la actualidad una de las principales prioridades dentro de la Unión Europea es la reducción de la exclusión social. En este sentido, los jóvenes al no encontrar un trabajo estable que les ayude a participar en la sociedad de manera activa se hallan en riesgo de exclusión. Para paliar dicha situación, desde la presente comunicación se expone una iniciativa nacida de los propios jóvenes que trata de crear una estructura físico-virtual que genere servicios, empleo y soporte a los nuevos emprendedores en materias de nuevas economías emergentes, para generar calidad de vida en la comunidad.

Palabras clave: Jóvenes, exclusión social, empleo, emprendedores, nuevas economías emergentes.

\section{YOUTH INITIATIVES AS A BASIS FOR INTEGRAL DEVELOPMENT. AREAS OF CO-WORKING AND NEW ECONOMIES: YOUTH HUB}

\section{Abstract}

Currently, one of the main priorities within the European Union is to reduce social exclusion. In this sense, youngsters unable to find a steady job to help them participate in society actively are at risk of exclusion. To remedy this situation, the present communication presents an initiative born from young 
people and trying to create a virtual physical structure that delivers services, employment and support to new entrepreneurs in the areas of new emerging economies, to generate quality of life in the community.

Key words: Youngsters, social exclusion, employment, entrepreneurs, new emerging economies.

\section{INTRODUCCIÓN}

Los constantes aumentos en las tasas de desempleo dificultan cada vez más, la supervivencia de los mecanismos de bienestar material y social. Además, un desempleo prolongado en el tiempo puede llevar a situaciones económicas muy difíciles de superar que en muchas ocasiones, sitúan a los afectados en riesgo de exclusión social. De manera tradicional, la población joven ha sido una de las categorias más vulnerables a los ciclos de crisis económicas, es decir, más propicias a una situación de desempleo sostenido o a la falta de empleo decente. En Italia, la situación laboral juvenil refleja un carácter particular respecto del resto de países de la Unión Europea, y prueba de ello es el evidente desequilibrio entre paro juvenil (15-34 años) y paro en personas mayores de 35 años. En este sentido, según cifras oficiales del Istat (Istituto Nazionale di Statistica), en el año 2012, mientras el paro juvenil rondaba cifras cercanas al $30 \%$ (situación récord en la historia del Istat), la desocupación en personas con edad superior a 35 años llegaba aproximadamente a un $7 \%$.

De entre todas las regiones italianas, Sicilia encabeza el grupo de los territorios que más padecen el fenómeno del desempleo juvenil, alcanzando cifras que rondan el $41,7 \%$ en menores de 30 años. Dicho valor llega a alcanzar el $51,3 \%$ si se contempla tan solo el grupo de edad de 15-24 años. En este sentido, como en el caso italiano en general, la desocupación en la población activa mayor de 35 años es mucho menor que la juvenil (12.9\%). Asimismo, para el área de implementación del proyecto Youth Hub, tanto la provincia de Siracusa cuenta con un paro juvenil del 40,4\%, en menores de 30 años y de tan solo $13,8 \%$ en mayores de 35 años; como la provincia de Catania cuenta con un $37,7 \%$ de paro juvenil, pero de tan solo un 10,3\% en mayores de 35 años. Sin embargo, todo ello demuestra que son los jóvenes quienes padecen de manera más directa el desempleo y por lo tanto, quienes tienen un mayor riesgo de padecer una exclusión social debido a no encontrar un trabajo estable que les ayude a participar en la sociedad de manera activa.

Además, la situación siciliana viene agravada por una crisis estructural mantenida a lo largo de su historia y que en la actualidad, se ve intensificada por factores como el aumento de la precariedad laboral. En dicha línea, la actual recesión no ha hecho más que intensificar dicha situación que afecta sobretodo a jóvenes cualificados que no encuentran buenas oportunidades laborales y 
que se ven obligados a aceptar trabajos precarios o en muchos casos, emigrar. De este modo, se desaprovecha uno de los recursos territoriales más valiosos con los que cuenta la isla: su capital humano y social.

Con todo, el desafío planteado respecto a la realidad de los jóvenes sicilianos requiere de una estrategia específica que de pie a un cambio desde la base, es decir, que surja de los propios afectados. Así, aprovechando sus aptitudes y poniéndolas en común con otros, conseguir formar una red que comience desde lo local. Dicha estrategia viene canalizada en la propuesta de un "Youth Hub" como centro de co-working, que actúa como ecosistema social, generando una comunidad de trabajo, donde jóvenes emprendedores o que solo tienen una idea empresarial se pueden conectar, crear sinergias y así, desarrollar e impulsar iniciativas en común.

\section{YoUTH HUB. UN ESPACIO PARA LA INNOVACIÓN SOCIAL Y LAS NUEVAS ECONOMÍAS}

En el actual momento de recesión, se hace más evidente la necesidad de crear, adaptar y difundir nuevas prácticas de tipo social que generen una dinámica de cambio en el territorio. En este sentido, los centros de co-working pueden actuar como el espacio propio donde diferentes grupos de personas se organizan y dan respuesta a sus retos. Son lugares donde trabajadores, jóvenes empresarios y micro empresas de diferentes sectores pueden compartir un mismo lugar de trabajo, ya sea físico o virtual, para desarrollar sus empresas, ya sea de forma individual o colaborativa. En un principio, los espacios de co-working se centraban en temáticas estrictamente profesionales, pero en la actualidad la diversidad de dichos espacios hace que además abarquen temas como el ocio, la cultura, la política o la gestión del territorio donde se localizan, reforzando aún más su sentido como ejemplos de innovación social. Prueba de ello fue el caso del co-working italiano Piano $C$, que ganó el primer premio del Social Innovation Tournament del EIB (European Investment Bank) en el año 2012. En esa línea, durante los últimos años, dichos espacios han ido ganando popularidad y se han venido desarrollando una gran variedad de casos en todo el mundo.

Así, uno de los ejemplos con mayor relevancia, debido tanto a su filosofía como a la difusión conseguida hasta el momento, es el caso de la red The HUB. The HUB es una comunidad internacional de emprendedores e innovadores sociales que inició en Islington (Londres) en el año 2005. Actualmente, cuenta con 31 espacios de coworking localizados y conectados por todo el mundo. Dicha comunidad está compuesta a su vez por más de 5000 miembros con perfiles que van desde emprendedores a freelance o artistas. En este sentido, tanto en España como en Italia existen algunos ejemplos que son referente tanto a nivel nacional como europeo. Así, en Italia existen diferentes ejemplos de The Hub, como Hub Roma, Milano, Trentino, Bari, Florencia o la iniciativa siciliana en Ortigia (Siracusa). Hoy en día, se desarrollan en él una gran diversidad 
de proyectos que incluyen temáticas que abarcan desde el cambio social y la sostenibilidad, a la tecnología, cultura y educación. Dicha comunidad es una evolución de los antiguos espacios co-working. Este nuevo modelo surge para apoyar, conectar e inspirar ideas en distintos territorios. Su principal objetivo es apoyar la incubación de empresas y el desarrollo de capacidades en los distintos eco-sistemas locales, teniendo en cuenta el impacto social.

En este contexto, aparece Youth Hub a un nivel de especificación y de evolución más alto. Youth Hub es un contexto de coworking gestionado por y para los jóvenes (tomando aquí la definición del Istat, que considera joven a aquella persona que tiene una edad comprendida entre 18-34 años). En dicho espacio (físico y virtual) los participantes pueden relacionarse, intercambiar ideas, colaborar y encontrar apoyo para desarrollar sus proyectos o promover otros proyectos en común. Todo ello en base a valores cooperativos que serán creados a partir de una comunidad de trabajo. Asimismo, otro aspecto característico de dicho espacio es su compromiso con el desarrollo del territorio donde se asienta. En este sentido, al ser un núcleo formado por los jóvenes del propio lugar, su compromiso por un desarrollo integrado y sostenible será visible.

Desde Youth Hub se apuesta por el sector de las nuevas economías emergentes. La experiencia y la actual crisis han demostrado como sólo la economía verde, economía del conocimiento, economía creativa o cultural, economía compartida y economía social están en grado de generar calidad de vida en la comunidad, bajo la base de un uso eficiente de los recursos materiales e inmateriales, la creación de puestos de trabajo y la sostenibilidad económica de las actividades productivas. En este sentido existen numerosos ejemplos reconocidos a nivel mundial como es el conocido caso de The Big Lemon, en Brighton y fundada en 2007 para desarrollar un nuevo servicio de autobús. La empresa aprovecha los autobuses desechados que son recuperados para hacerlos funcionar con aceite de cocina de bares y restaurantes, una vez reciclado. Entre las ventajas de esto, se encuentran la reducción de dos tercios de emisiones a la atmósfera y la reducción del precio del servicio de transporte. Por ello, Youth Hub apuesta por este sector económico con el fin de realizar un modelo de territorio vivible, viable y ecuo, sea para las generaciones presentes que para las futuras. En definitiva, el objetivo del Youth Hub es generar una red de jóvenes emprendedores que se comprometan en la cooperación, las nuevas economías emergentes, el desarrollo local sostenible, en el feedback urbano-rural y en la innovación territorial como estrategia para superar la actual crisis.

El proyecto Youth Hub está compuesto de tres espacios de coworking obteniendo una dimensión sub-regional localizada en la región sudoriental de la isla de Sicilia. En primer lugar está Youth Hub Motta Sant'Anastasia situado en las faldas del Etna (municipios de Motta Sant'Anastasia, Misterbianco, Camporotondo, Belpasso y San Pietro Clarenza), que cuenta con un total de 46.438 jóvenes entre 15 y 30 años (47\% de la población total), en 
segundo lugar tenemos Youth Hub Floridia que pasa por la zona occidental de la provincia de Siracusa (municipios de Floridia, Solarino, Canicattini Bagni, Palazzolo Acreide, Sortino, Ferla, Cassaro), que cuenta con un total de 20.643 jóvenes $(34,5 \%)$, y llegando hasta el extremo sur de Sicilia y de la provincia de Siracusa (municipios de Noto, Avola, Pachino, Portopalo di Capo Passero y Rosolini), que cuenta con un total de 34.320 jóvenes $(33,2 \%)$ tenemos Youth Hub Noto. El objetivo es desarrollar un ecosistema social donde los jóvenes emprendedores puedan conectarse para aprovechar sus sinergias y configurar, crear y transformar sectores y territorios competitivos en manera creativa. Así, al mismo tiempo, ofrecer los instrumentos, impulsos y ayuda que tantas veces los jóvenes no tienen a la hora de emprender.

\section{Youth Hub Network COMo EXPeriencia DE tRabajo EN RED}

Los tres Youth Hub trabajarán en red, compartiendo así costes y beneficiándose de las economías de escala que surjan entre ellos. Será una red de tipo articulado, donde cada servicio y cada profesional conocerá las funciones y las competencias de los otros servicios y profesionales. Otras características de la red será la valoración igualitaria y la reciprocidad. En este sentido, respecto de la valoración igualitaria en el caso de Youth Hub Network, los participantes han elegido al Youth Hub Floridia como jefe de fila del proyecto debido a su mayor experiencia en el trabajo en red y que cuenta con una mayor capacidad técnica entre sus jóvenes. Sin embargo, a través del concepto "valoración igualitaria" se pretende destacar que no habrá un único centro, ni un eje central dentro de la red, sino que habrá una rotación de roles (la misma actuación la pueden realizar varios miembros y son variables) y una jerarquía funcional (cada uno puede dominar un tema en concreto). Además, Youth Hub Network pretende implicar a los territorios limítrofes para que puedan disfrutar de los resultados del proyecto en sus contextos territoriales, y así, extender la red a nivel regional a medio-largo plazo.

La propuesta de Youth Hub Network ofrece acceso a todos los jóvenes entre 18 y 34 años, sin excluir a ninguna persona que quiera participar y que tenga otra edad, con el objetivo de conseguir una participación activa que les implique y les facilite su inclusión como ciudadanos activos dentro de la sociedad. Además, otra prioridad importante que será satisfecha es la de ofrecer el mismo acceso y participación a los jóvenes con menores oportunidades por motivos económicos, culturales, sociales o de discapacidad. En este sentido, desde Youth Hub Network se trabajará por tomar todas las medidas necesarias para conseguir la inclusión social, sobretodo incentivando la ciudadanía activa y la ocupación de los jóvenes con menores oportunidades "on-the-job", contribuyendo a la cohesión dentro de la comunidad, y a la aplicación de la estrategia para la inclusión de la Unión Europea. 
Además, el proyecto promueve el compromiso de los jóvenes sobre un crecimiento más inclusivo, debido a que afronta el problema de la desocupación juvenil y mira por la participación activa de los jóvenes desocupados en la sociedad, garantizando el acceso a todas las oportunidades que les ofrece. En esta línea, Youth Hub Network dedica un particular interés por implicar a jóvenes inmigrantes, jóvenes discapacitados y jóvenes que pertenecen a otras categorías en desventaja, ya presentes en la red y que estimulan el espíritu de la iniciativa, su creatividad y su vocación a la emprenditorialidad.

El proyecto está dirigido a los jóvenes que tengan la voluntad de desarrollar su negocio sobre la base de la inclusión de las nuevas economías y la innovación en su estrategia, productos, servicios y procesos de trabajo. Para ello, Youth-Hub Network se centrará en mejorar las competencias, aumentando los recursos a disposición de los jóvenes en materia de empleo, de manera que puedan recibir un apoyo adecuado y dónde los servicios que se ofrezcan sean de calidad respetando todos sus derechos; poner en práctica programas de formación y desarrollo de las competencias empresariales; apoyar a las empresas para que puedan conservar su fuerza de trabajo por medio de mecanismos bien concebidos que se pongan en práctica a través del diálogo social y la negociación colectiva (entre estos mecanismos se incluye el trabajo compartido); apoyar la creación de empleo en los sectores dedicados a las nuevas economías reconociendo la contribución de las PYMES y de las microempresas a la creación de empleo; establecer entornos normativos que sean favorables y propicios a la generación de empleo a través de la creación y el desarrollo de empresas sostenibles.

Por lo tanto Youth Hub Network buscará favorecer un proceso de crecimiento emprenditorial y ocupacional juvenil, a través de un método participativo y democrático, como instrumento activo de colaboración e inclusión social, y mediante la difusión y el uso de ejemplos derivados de las nuevas economías como herramientas para abordar y superar la actual crisis cíclica. Todo ello a partir de las sinergias establecidas como consecuencia del networking que generará una red de jóvenes emprendedores comprometidos a trabajar en la cooperación, las nuevas economías emergentes, el desarrollo local sostenible, el "feedback" urbano-rural y la innovación territorial como estrategia para superar la crisis actual.

\section{Comunidad DE TRABAJO PARTICIPATIVA E INCLUSIVA}

Youth Hub Network surge a partir una idea compartida entre un grupo informal de jóvenes apoyados por el Lead thematic expert Sergio Campanella. En este sentido, se trata de un grupo interdisciplinar muy sensibilizado con las cuestiones del desarrollo territorial y las problemáticas juveniles. No obstante, entre los cinco jóvenes promotores se encuentran perfiles que abarcan desde 
la Geografía y la Sociología, a la Psicología y el Desarrollo Local. Asimismo, la colaboración y la función del politólogo, doctor Sergio Campanella como coach durante el proyecto, facilitará la gestión de funciones como la consolidación del grupo, la autogestión del espacio, el proceso de aprendizaje o la organización estratégica, entre otras.

Hasta el momento se han realizado las actividades pertinentes a la organización y puesta en marcha de cada Youth Hub. Es decir, en primer lugar implicar tanto a la administración pública, como a los diferentes agentes locales del territorio. En segundo lugar se han realizado cuatro encuentros participativos en cada uno de los ayuntamientos. Donde la asistencia y participación total ha sido de 92 jóvenes con edades comprendidas entre 18 y 30 años. De este grupo informal de jóvenes que participará en el proyecto, 48 están en riesgo de exclusión, ya sea por sus dificultades económicas o porque además se le unen otros factores como ser discapacitado o inmigrante. Durante los diferentes encuentros participativos llevados a cabo durante la fase preparatoria, se desarrollaron dinámicas de grupo muy conocidas a nivel europeo como EASW ${ }^{\circledR}$, World Café, De Bono's Thinking Hats, Gulliver's Maps, etc. El objetivo de dichas dinámicas fue empoderar a los participantes, facilitar la des-inhibición, promover el conocimiento y la cohesión del grupo, facilitar el debate y el libre intercambio de ideas y opiniones entre participantes sobre los diferentes aspectos relativos al Youth Hub.

Otro aspecto a tener en cuenta hasta ahora, es el número de partners que participan en el Youth Hub y que ayudarán poniendo a disposición del proyecto recursos tangibles e intangibles. Dichos partners son: los ayuntamientos de Floridia (SR), Motta Sant' Anastasia (CT), Noto (SR), San Pietro Clarenza (CT), Canicattini Bagni (SR) y Solarino (SR), que pondrán a la disposición del Hub recursos propios como su personal, o en algunos casos espacios confiscados a la Mafia y otro tipo de recursos técnicos; la Agencia Jóvenes EURODESK de Catania, ofrecerá numerosa información a los jóvenes sobre aspectos como la movilidad, la formación o el trabajo; el Instituto Técnico Económico "De Felice Olivetti" de Catania, aportará servicios de orientación y apoyo a los jóvenes; la asociación LIFE Onlus, Metamorfosis y "Padre Pio" que se dedicarán a la promoción de actividades enfocadas a jóvenes discapacitados; la asociación Eminescu que trabajará por la integración del colectivo de inmigrantes; el GAL Eloro que apoyará al Youth Hub aportando la colaboración de parte de su capital humano.

En cuanto a la difusión, a día de hoy se ha trabajado por conseguir una gran difusión de la red. Así, en cuanto a las redes sociales, se han creado tres perfiles en Facebook, que actualmente cuentan con alrededor de 800 seguidores y donde la participación es muy fluida. Por otro lado, son varias las publicaciones que han aparecido en la prensa local (Giornale di Sicilia) hablando de esta iniciativa juvenil. Además, a fecha de 15 de mayo, se presentó la candidatura del 
proyecto "YOUTH HUB NETWORK" en el segundo torneo sobre innovación social del EIB (European Investment Bank). Por último, otro ejemplo sería la presente comunicación para este coloquio organizado por el Grupo de Trabajo de Desarrollo Local de la Asociación de Geógrafos Españoles en la Universidad de Alicante consiguiendo una difusión transnacional.

En definitiva, se espera que el impacto del proyecto Youth Hub Network sobre el tejido socioeconómico local sea notable, en cuanto a que sus actividades no están circunscritas exclusivamente a los participantes de la actividad. Al contrario, se prevé agrupar al mayor número posible de gente, sean de la propia comunidad, sean de territorios limítrofes y prueba de ello, el importante esfuerzo que se está llevando a cabo por conseguir implicar al máximo número de personas y partners. En realidad, Youth Hub Network, pese a estar localizado en un área concreta, estará abierto a crear flujos y sinergias con otros territorios para así combatir problemáticas comunes. No obstante, el proyecto de Network está encuadrado en una prospectiva a largo plazo y programado para producir un efecto multiplicador y un impacto sostenible que sean tangibles y durables.

BIBLIOGRAFÍA

EIB (2013) Social Innovation Tournament. http://institute.eib.org/programmes/ social/social-innovation-tournament/ [consulta: 12/04/2013]

ESPON - ATTREG (2010/2012) - Attractiveness of European Regions and Cities for Residents and Visitors http://www.espon.eu/main/Menu_Projects/ Menu_AppliedResearch/attreg.html, [consulta: 24/03/2013]

Istat (2012): Tasso di dissocupazione -livello regionale. http://www.istat.it/it/ istituto-nazionale-di-statistica [consulta: 11/03/2013]

Istat (2012): Tasso di dissocupazione- livello provinciale. http://www.istat.it/it/ istituto-nazionale-di-statistica [consulta: 11/03/2013]

The HUB Barcelona. http://hub-barcelona.net/ , [consulta: 26/03/2013]

The HUB Madrid https://madrid.the-hub.net/ , [consulta: 26/03/2013]

URBAN N.O.S.E. (Towards and urban economic system of Social Incubators http://urbact.eu/en/projects/human-capital-entrepreneurship/urban-nose/homepage/ , [consulta: 24/03/2013]

Youth Hub Floridia https://www.facebook.com/youthhub.floridia?fref=ts , [consulta: 15/04/2013]

Youth Hub Motta Sant'Anastasia https://www.facebook.com/youthhub.mottas. anastasia?fref=ts , [consulta: $15 / 04 / 2013$ ]

Youth Hub Noto https://www.facebook.com/noto.youthhub.3?fref=ts , [consulta: $15 / 04 / 2013$ ] 